\title{
Grand challenges in computational physiology and medicine
}

\author{
Raimond L. Winslow* \\ Department of Biomedical Engineering, Institute for Computational Medicine, The Johns Hopkins University School of Medicine and Whiting School of Engineering, \\ Baltimore, MD, USA \\ ${ }^{*}$ Correspondence: rwinslow@jhu.edu
}

\section{OVERVIEW}

The nature of basic biological research has been transformed during the past decade. This transformation has been driven in part by development of new technologies for high-throughput data acquisition that now make it possible to sequence genomes (Metzker, 2009), and to measure RNA (Wang et al., 2009) and protein (Maerkl, 2011) expression levels with ever increasing accuracy and lower cost. These extraordinary achievements have contributed to advances in genetic medicine (Amberger et al., 2009) and the discovery of gene and protein signatures of disease (Wood et al., 2007; Hanash and Taguchi, 2010; Addona et al., 2011; Cancer Genome Atlas Research Network, 2011; Heidecker et al., 2011; Majewski and Bernards, 2011). There is, however, growing recognition that the tabulation of molecular building blocks from which biological systems are composed is not sufficient for understanding the functional properties of these systems. Indeed, function does not exclusively arise from vertical integration beginning at the level of either gene, RNA, or protein. Rather, it is becoming clear that the emergent, integrative behaviors of biological systems result from complex interactions both within and across different hierarchical levels of biological organization. As but one example, both RNA (Morris, 2011), proteins (Licatalosi and Darnell, 2010), electrical and mechanical function at the level of cells and tissue (Dolmetsch, 2003; Barlow et al., 2006; Gundersen, 2011), and external cues including environmental factors (Jaenisch and Bird, 2003; Laird, 2010) can all feed-back to regulate gene expression. This coupling between different levels of biological organization has been referred to by Noble (2006, $2008,2009)$ as upward and downward pathways of causation. A consequence is that function cannot be considered to reside at any one level. Rather, function arises from the integrated behavior of the overall system, a concept that can be viewed as a new "central principle of biology." Because of the inherent complexity of real biological systems, attempts to intuit their behavior using "mental models" often fail. Instead, the development and analysis of computational models based directly on experimental data is proving to be a powerful approach. We refer to this as Computational Physiologymodeling directed at achieving a quantitative understanding of the normal functional processes of an organism, organ, or system. Such models provide the foundation for understanding perturbed physiological function in disease - an approach we refer to as Computational Medicine.

Computational modeling is now a core component of many scientific disciplines including physics, chemistry, economics, psychology, public health, and most recently biology. One power of computational modeling is that the scale of models that can be formulated and analyzed far exceeds anything that can be done using more traditional paper-and-pencil mathematical approaches. This is particularly key in the extraordinarily complex realm of physiology and medicine. There is no question that understanding how function emerges as a consequence of connectivity both within and across the organizational levels of molecules, pathways, cells, tissues, and organs is the next major frontier of biomedical science, and computation will play a key role in achieving this understanding. The impact of computational medicine on society will be particularly profound. Our understanding of disease has only scratched the surface, and we will never have the ability to develop effective, personalized therapies unless we can truly understand the link between molecules and phenotypes at a quantitative, mechanistic level.

What should we expect of computational models in physiology and medicine? First, models should have a firm basis in experimental data. To gain deep insights into physiological function, models should be mechanistic rather than formulated solely in terms of statistical inference. Models must be able to reconstruct the data on which they are based, and this should be demonstrated clearly when publishing models. Second, models must make predictions (hypotheses) that can be tested experimentally. Model predictions may or may not be supported by results from the experimental studies they motivate. In either case, by using models to generate testable hypotheses about system function, we can better appreciate what is and is not known about the mechanisms underlying function. All models will fail at some point. Such failures can be highly informative, and modelers must be willing to communicate both the successes and failures of their models to the community. By following this approach, a closed loop can be established in which model-based hypothesis generation motivates experimental testing and model refinement. Third, a goal of modeling should be to fit into the paradigm of upward and downward pathways of causality. That is, if function does not solely reside at any one level of biological organization, then the process of developing and refining models based on data obtained at a specific hierarchical level should build toward the goal of identifying and accurately capturing the behavior of those variables which are the connection points between levels. This is the only way in which integrative (often referred to as multi-scale) models that provide deep insights into physiological function will ever be developed. Finally, models of disease, which will likely take the form of normal physiological systems models operating in a different parameter regime (Belair et al., 1995; Huang et al., 2009), must not only be reconstructive and predictive, they must guide the way to improved therapies.

\section{GRAND CHALLENGES}

Achieving the expectations regarding mechanistically based computational models of physiological function in health and disease outlined above is itself a grand 
challenge. It is easy to say that the failure of a model to predict experimental outcomes should drive model refinement and further experimental testing. However, in many instances discrepancies between model predictions and experimental outcomes may suggest that additional biological processes must be incorporated into the model, or that some existing model component needs more precise characterization. Doing so may require the development of new experimental approaches, which can often be a daunting task. An example from the field of cardiac electrophysiology was the realization that accurate characterization and modeling of voltage-dependent membrane currents underlying electrical excitability of cardiac myocytes was not going to be possible using intracellular recordings obtained from small tissue samples (circa 1960s-1970s) due, among other things, to an inability to space-clamp cells and to prevent accumulation of potassium in the extracellular space during repeated stimulation. Rather, this characterization required that recordings be performed in isolated myocytes, a technique that was not perfected until the early 1980s. It is also easy to say that experimental results must drive the refinement of models. However, this can often be extremely challenging when models incorporate many different interacting biological processes. It is not enough that the revised model explain the new experimental data, it must also be shown that as models are refined, they continue to reconstruct the legacy data on which they were based. Techniques such as sensitivity analysis (Marino et al., 2008) or use of minimization algorithms to estimate model parameters that yield a best fit to large constraining data sets (Lillacci and Khammash, 2010) can sometimes help. However there is no guarantee that these methods will converge to a global minima when dealing with high-dimensional models.

These general aspects of physiological modeling are certainly grand challenges, however, there are also emerging research areas that deserve special mention. Noting these areas is not intended in any way to limit the scope of articles that are appropriate for this journal. Rather, these are the author's views regarding grand challenges that cut across many different areas of computational physiology and medicine.

\section{D SYSTEMS BIOLOGY}

There is compelling evidence that spatial co-location of proteins that interact with one another over nanometer length scales to perform biological functions is a common biological design motif. These regions of co-location are often referred to as "nano-domains." One example is the process of calcium-induced calcium-release (CICR) in the cardiac myocyte (Winslow and Greenstein, 2011). The structural basis of this process is that voltage-gated L-type calcium (Ca) channels (LCCs) in the cell membrane are located in direct opposition to Ca-binding Ca release channels (ryanodine receptors, RyRs) in the closely opposed junctional sarcoplasmic reticulum (JSR) membrane. These regions of opposition are known as dyads, and have depth (the bounding distance between LCCs and RyRs) and diameter of $\sim 10$ and $50-100 \mathrm{~nm}$, respectively. Opening of LCCs and influx of Ca triggers opening of RyRs and release of Ca into the small volume of the dyad. As Ca concentration rises, Ca induces inactivation of the LCCs, establishing a coupled feedforward activation and feed-back inactivation process. Given the spatial co-location of LCCs and RyRs within this nano-domain, they function as a coupled unit, with their interaction being mediated by $10 \mathrm{~s}$ of $\mathrm{Ca}$ ions, making it a noisy process. They are an example of a "protein machine" (Alberts, 1998), and their functional behavior at the nanoscale level ultimately leads to ensemble behavior at the macroscopic level that drives contraction of the heart. Even subtle disruptions of the spatial arrangement of RyRs and LCCs can have significant impact on CICR at the cellular level (Tanskanen et al., 2007). Such changes occur in heart failure, likely contributing to altered function of this protein machinery, and understanding of this altered function will be a key step forward in developing new therapeutic approaches to treating heart failure (Anderson and Mohler, 2009). Other examples of spatially dependent protein interactions within nano-domains abound. Lipid raft Ras nanoclusters with diameter of 6-12 nm, consisting of active Ras, the MAPK module and scaffolding proteins form transiently $(\sim 400 \mathrm{~ms})$ to produce a digital burst of active ERK (Harding and Hancock, 2008). Ca signaling within nano-domains localized to dendritic spines is known to activate downstream effectors such as CaMKII and cAMP-dependent pathways (Higley, 2008). To date, systems biology has largely explored "flat," graph-based patterns of component interconnections. However, the above examples show that to understand integrated systems behavior, we must consider how interacting networks of genes, proteins, membranes, and filaments are arranged in 3D space, how this determines their interaction dynamics, and how these spatial relations are altered in disease. A grand challenge is to develop methods for modeling the function of these protein machines at the nanoscale level, and mathematical and computational approaches for simplifying these models so that they may be incorporated within a multi-scale modeling framework, as described below.

\section{MULTI-SCALE MODELING}

Multi-scale modeling refers to the process of modeling physiological function across multiple scales of biological organization. The power of multi-scale modeling is that it can provide insights into how system properties at the molecular level map to function at a more macroscopic level. Thus, it has the potential to form the long sought bridge between genotype and phenotype. Application areas include modeling the electromechanical function of the heart from ion channels to cells and organ (Trayanova and Rice, 2011), angiogenesis (Qutub et al., 2009), tumor growth (Hatzikirou et al., 2011), and bone remodeling (Webster and Müller, 2011), to name a few. Without question, the most ambitious effort in this area is the Virtual Physiological Human project (Viceconti et al., 2008), the goal of which is to establish a methodological and technological framework for studying the human body as a single complex system.

Multi-scale modeling poses numerous mathematical and computational challenges. Typically, they arise from the different spatio-temporal scales used to model at one level versus another, coupled with the need to combine these scales. In such cases, an appropriate succession of mathematical approximations and computational approaches must be developed in order to model across levels. An example is the work of Tanskanen and Winslow (2006) in developing multi-scale models of CICR. Since signaling in nano-domains is often mediated by small numbers of molecules, well outside the regime of the laws 
of mass-action, the starting point of this work was development of a model describing the motion of individual Ca ions in the cardiac dyad. To do this, the Fokker-Plank equation specifying the time-evolution of the probability that $r \mathrm{Ca}$ ions were at a given dyadic positions at time $t$ was solved by discretizing this equation, yielding a large Markov chain modeling ion movement driven by Brownian motion in a potential field. This model was further simplified by assuming that $\mathrm{Ca}$ ions were independent and that their binding to buffers, receptors, and membranes was in equilibrium. Conditioning on a configuration of Ca sources (RyRs and LCCs) then reduced the FPE for individual ion locations and numbers to a reaction-diffusion equation. Since Ca diffusion on $\mathrm{nm}$ length scales was several orders of magnitude faster than channel gating, $\mathrm{Ca}$ ion density in the dyad equilibrated rapidly in each LCC and RyR gating state, reducing the reaction-diffusion equation to a relatively low-dimensional system of ordinary differential equations. Integration of these equations into cell models allowed realistic description of CICR in larger scale tissue and whole heart models. Therefore, the key to this multi-scale modeling approach was a series of careful approximations regarding independence of signaling molecules and successive mathematical approximations based on time-scale separation. Mathematical approaches to multi-scale model simplification are likely to vary from problem to problem, and with the specific modeling approaches employed at each level. For example, the concepts of tunable resolution and fragmentation are used with rules-based modeling, and help to eliminate the combinatorial complexity of network models (Harmer, 2009; Harmer et al., 2010). In addition, multi-scale modeling often requires development of new, fast numerical methods (for example, fast stochastic simulation methods; Xu and Cai, 2008), development of specialized highperformance computing approaches utilizing large-scale parallelization (Markram, 2006; Reumann et al., 2008), and/or specialized processor designs (Christley et al., 2010). Thus, significant challenges remain to be overcome before the use of multi-scale models becomes more widespread. Case studies in new application areas that test new theories, algorithms, and computational methods will be key to building a general approach to multi-scale modeling of physiological system function in health and disease.

\section{PATIENT-SPECIFIC MODELING}

The goal of patient-specific modeling (Neal and Kerckhoffs, 2010) is to develop computational models of pathophysiology using data from an individual patient. It has the potential to guide the delivery of treatment tailored to individual needs. Patient-specific modeling is being applied to predicting risk of vertebral osteoporotic fracture (Travert et al., 2011), 3D finite-element modeling of bone grafts (Diederichs et al., 2008), computational modeling of intra-aneurism hemodynamics (Castro et al., 2006), electromechanical modeling of the failing human heart (Aguado-Sierra et al., 2011), and tumor growth (May et al., 2011), among other applications. Most of this modeling is driven by the ever expanding ability to collect high spatio-temporal resolution patient image data that can in turn be used to generate models of the relevant structures. While the field holds great promise, it is important to select applications in which the biological properties that are critical to the predictive value of the modeling can be measured. For example, it is possible to image the geometry and motion of the heart in a patient with non-ischemic cardiomyopathy and then fit a generic finite-element mechanics model to these data. However, it is also known that there is extensive spatial remodeling of the activity of key proteins controlling electrical excitability, Ca dynamics, signaling pathways, and metabolic processes of cardiac myocytes in the failing heart. These features may be important for modeling cardiac electromechanical function in a patient-specific manner. There is as yet no method for measuring this remodeling in the individual patient. For these reasons, testing approaches for patient-specific modeling in carefully selected animal models is likely to be important for assessing the validity of these approaches.

\section{INFORMATICS IN COMPUTATIONAL PHYSIOLOGY AND MEDICINE}

Almost from its inception, the highthroughput genomics community has had a culture of sharing data. Unfortunately, this culture does not yet exist within the broader physiological community. Despite the fact that the National Institutes of Health requires that data obtained in grants with more than $\$ 500,000$ in direct costs per year be shared, this is almost never done. The barrier is both cultural and technical. Physiological data is diverse and complex, and the software tools for managing these data, and ontology for describing physiological experiments and data have not yet been developed. Doing so is critically important, as these data, collected at the cost of hundreds of billions of tax-payer dollars, are quite literally being lost. Developing the tools for managing, annotating, searching, and sharing physiological data is essential for the advancement of the disciplines of physiology and medicine, and is necessary for the development of quantitative models of physiological function in health and disease. One first step has been the recent proposal of a minimum information reporting standard for a cardiac electrophysiological experiment (Quinn et al., 2011) and a neuroscience investigation (Gibson et al., 2009).

There have been recent advances in creating software tools for disseminating computational models. Model sharing has been a difficult thing to achieve. It is not sufficient that model equations be published in the peer-reviewed literature because in the majority of instances, models are simply too complex to avoid errors in either equations or parameters. The CellML (Beard et al., 2009), Systems Biology Markup Language (SBML; Hucka et al., 2003), and NeuroML (Gleeson et al., 2010) have been developed as a way of addressing this problem. These languages, which are subsets of the eXtensible Markup Language (XML), support the description of model equations and parameters in machine readable form. Of course, CellML, SBML, and NeuroML model description documents must be validated on creation, but once they have been, they can be disseminated in an errorfree way and input to a number of different simulation tools for execution (Alves and Antunes, 2006; Keating et al., 2006). A number of different model repositories such as CellML.org and the BioModels Database (Li et al., 2010) have been developed that allow users to search for and download XML model descriptions. Currently, these model description languages are able to capture biological systems models that may be cast in the form of reaction networks. Developing methods by which these model 
descriptions can be composed into larger, potentially multi-scale models is a promising direction for future research.

\section{CONCLUSION}

We are poised at an exciting moment in the life sciences, one at which the fruits of reductionist biology and the continuing consequences of Moore's Law and it's impact on computational science are coming together to drive the re-birth of integrative physiology and the creation of a new, quantitative, modeling-based approach to medicine. As the vanguards of this new effort, we must all work together to assemble and understand the Physiome. Frontiers in Computational Physiology and Medicine will provide the community a unique venue to help achieve this goal.

\section{REFERENCES}

Addona, T.A., Shi, X., Keshishian, H., Mani, D. R., Burgess, M., Gillette, M. A., Clauser, K. R., Shen, D., Lewis, G. D., Farrell, L.A., Fifer, M.A., Sabatine, M. S., Gerszten, R. E., and Carr, S.A. (2011). A pipeline that integrates the discovery and verification of plasma protein biomarkers reveals candidate markers for cardiovascular disease. Nat. Biotechnol. 29, 635-643.

Aguado-Sierra, J., Krishnamurthy, A., Villongco, C., Chuang, J., Howard, E., Gonzales, M. J., Omens, J., Krummen, D. E., Narayan, S., Kerckhoffs, R. C. P., and McCulloch, A. D. (2011). Patient-specific modeling of dyssynchronous heart failure: a case study. Prog. Biophys. Mol. Biol. 1-9.

Alberts, B. (1998). The cell as a collection of protein machines: preparing the next generation of molecular biologists. Cell 92, 291-294.

Alves, R., and Antunes, F. (2006). Tools for kinetic modeling of biochemical networks. Nat. Biotechnol. 24, 667-672.

Amberger, J., Bocchini, C. A., Scott, A. F., and Hamosh, A. (2009). McKusick's online Mendelian inheritance in man (OMIM(R)). Nucleic Acids Res. 37, D793-D796.

Anderson, M. E., and Mohler, P. J. (2009). Rescuing a failing heart: think globally, treat locally. Nat. Med. $15,25-26$.

Barlow, C., Rose, P., Pulver-Kaste, R., and Lounsbury, K. (2006). Excitation-transcription coupling in smooth muscle. J. Physiol. 570, 59-64.

Beard, D. A., Britten, R., Cooling, M. T., Garny, A., Halstead, M. D. B., Hunter, P. J., Lawson, J., Lloyd, C. M., Marsh, J., Miller, A., Nickerson, D. P., Nielsen, P. M., Nomura, T., Subramanium, S., Wimalaratne, S. M., and Yu, T. (2009). CellML metadata standards, associated tools and repositories. Philos. Transact. A Math. Phys. Eng. Sci. 367, 1845-1867.

Belair, J., Glass, L., An Der Heiden, U., and Milton, J. (1995). Dynamical disease: identification, temporal aspects and treatment strategies of human illness. Chaos 5, 1-7.

Cancer Genome Atlas Research Network. (2011). Integrated genomic analyses of ovarian carcinoma. Nature 474, 609-615.

Castro, M. A., Putman, C. M., and Cebral, J. R. (2006). Patient-specific computational fluid dynamics mod- eling of anterior communicating artery aneurysms: a study of the sensitivity of intra-aneurysmal flow patterns to flow conditions in the carotid arteries. AJNR Am. J. Neuroradiol. 27, 2061-2068.

Christley, S., Lee, B., Dai, X., and Nie, Q. (2010). Integrative multicellular biological modeling: a case study of $3 \mathrm{D}$ epidermal development using GPU algorithms. BMC Syst. Biol. 4, 107. doi: 10.1186/1752-0509-4-107

Diederichs, G., Seim, H., Meyer, H., Issever, A. S., Link, T. M., Schroder, R. J., and Scheibel, M. (2008). CT-based patient-specific modeling of glenoid rim defects: a feasibility study. AJR Am. J. Roentgenol. 191, 1406-1411.

Dolmetsch, R. (2003). Excitation-transcription coupling: signaling by ion channels to the nucleus. Sci. Signal. 2003, 4.

Gibson, F., Overton, P., Smulders, T., and Schultz, S. (2009). Minimum information about a neuroscience investigation (MINI): electrophysiology. Available at: http://hdl.handle.net/10101/npre.2009.1720.2

Gleeson, P., Crook, S., Cannon, R. C., Hines, M. L., Billings, G. O., Farinella, M., Morse, T. M., Davison, A. P., Ray, S., Bhalla, U. S., Barnes, S. R., Dimitrova, Y. D., and Silver, R. A. (2010). NeuroML: a language for describing data driven models of neurons and networks with a high degree of biological detail. PLoS Comput. Biol. 6, e1000815. doi: 10.1371/journal. pcbi. 1000815

Gundersen, K. (2011). Excitation-transcription coupling in skeletal muscle: the molecular pathways of exercise. Biol. Rev. Camb. Philos. Soc. 86, 564-600.

Hanash, S., and Taguchi, A. (2010). The grand challenge to decipher the cancer proteome. Nat. Rev. Cancer 10, 652-660.

Harding, A. S., and Hancock, J. F. (2008). Using plasma membrane nanoclusters to build better signaling circuits. Trends Cell Biol. 18, 364-371.

Harmer, R. (2009). Rule-based modelling and tunable resolution. Electron. Proc. Theor. Comput. Sci.9, 65-72.

Harmer, R., Danos, V., Feret, J., Krivine, J., and Fontana, W. (2010). Intrinsic information carriers in combinatorial dynamical systems. Chaos 20, 037108.

Hatzikirou, H., Chauviere, A., Bauer,A. L., Leier, A., Lewis, M. T., Macklin, P., Marquez-Lago, T. T., Bearer, E. L., and Cristini, V. (2011). Integrative physical oncology. Wiley Interdiscip. Rev. Syst. Biol. Med. doi: 10.1002/ wsbm. 158

Heidecker, B., Kittleson, M. M., Kasper, E. K., Wittstein, I. S., Champion, H. C., Russell, S. D., Hruban, R. H., Rodriguez, E. R., Baughman, K. L., and Hare, J. M. (2011). Transcriptomic biomarkers for the accurate diagnosis of myocarditis. Circulation 123, 1174-1184.

Higley, M. (2008). Calcium signaling in dendrites and spines: practical and functional considerations. Neuron 59, 902-913.

Huang, S., Ernberg, I., and Kauffman, S. (2009). Cancer attractors: a systems view of tumors from a gene network dynamics and developmental perspective. Semin. Cell Dev. Biol. 20, 869-876.

Hucka, M., Finney, A., Sauro, H. M., Bolouri, H., Doyle, J. C., Kitano, H., Arkin, A. P., Bornstein, B. J., Bray, D., Cornish-Bowden, A., Cuellar, A. A., Dronov, S., Gilles, E. D., Ginkel, M., Gor, V., Goryanin, I. I., Hedley, W. J., Hodgman, T. C., Hofmeyr, J. -H., Hunter, P. J., Juty, N. S., Kasberger, J. L., Kremling, A., Kummer, U., Le Novère, N., Loew, L. M., Lucio, D., Mendes, P., Minch, E., Mjolsness, E. D., Nakayama, Y., Nelson, M. R., Nielsen, P. F., Sakurada, T., Schaff, J. C., Shapiro, B. E., Shimizu, T. S., Spence, H. D., Stelling, J., Takahashi, K., Tomita, M., Wagner, J.,
Wang, J., and SBML Forum. (2003). The systems biology markup language (SBML): a medium for representation and exchange of biochemical network models. Bioinformatics 19, 524-531.

Jaenisch, R., and Bird,A. (2003). Epigenetic regulation of gene expression: how the genome integrates intrinsic and environmental signals. Nat. Genet. 33, 245-254.

Keating, S. M., Bornstein, B. J., Finney, A., and Hucka, M. (2006).SBMLToolbox: an SBML toolbox for MATLAB users. Bioinformatics 22, 1275-1277.

Laird, P.W. (2010). Principles and challenges of genomewide DNA methylation analysis. Nat. Rev. Genet. 11, 191-203.

Li, C., Donizelli, M., Rodriguez, N., Dharuri, H., Endler, L., Chelliah, V., Li, L., He, E., Henry, A., Stefan, M. I., Snoep, J. L., Hucka, M., Le Novère, N., and Laibe, C. (2010). BioModels Database: an enhanced, curated and annotated resource for published quantitative kinetic models. BMC Syst. Biol. 4, 92. doi: 10.1186/1752-0509-4-92

Licatalosi, D. D., and Darnell, R. B. (2010). RNA processing and its regulation: global insights into biological networks. Nat. Rev. Genet. 11, 75-87.

Lillacci, G., and Khammash, M. (2010). Parameter estimation and model selection in computational biology. PLoS Comput. Biol.6, e1000696. doi: 10.1371/journal. pcbi.1000696

Maerkl, S. J. (2011). Next generation microfluidic platforms for high-throughput protein biochemistry. Curr. Opin. Biotechnol. 22, 59-65.

Majewski, I. J., and Bernards, R. (2011). Taming the dragon: genomic biomarkers to individualize the treatment of cancer. Nat. Med. 17, 304-312.

Marino, S., Hogue, I. B., Ray, C. J., and Kirschner, D. E. (2008). A methodology for performing global uncertainty and sensitivity analysis in systems biology. J. Theor. Biol. 254, 178-196.

Markram, H. (2006). The blue brain project. Nat. Rev. Neurosci. 7, 153-160.

May, C. P., Kolokotroni, E., Stamatakos, G. S., and Büchler, P. (2011). Coupling biomechanics to a cellular level model: an approach to patient-specific image driven multi-scale and multi-physics tumor simulation. Prog. Biophys. Mol. Biol. 1-7.

Metzker, M.L. (2009). Sequencing technologies - the next generation. Nat. Rev. Genet. 11,31-46.

Morris, K. (2011). The emerging role of RNA in the regulation of gene transcription in human cells. Semin. Cell Dev. Biol. 22, 351-358.

Neal, M. L., and Kerckhoffs, R. (2010). Current progress in patient-specific modeling. Brief. Bioinform. 11, 111-126.

Noble, D. (2006). Systems biology and the heart. Biosystems 83, 75-80.

Noble, D. (2008). Claude Bernard, the first systems biologist, and the future of physiology. Exp. Physiol. 93, 16-26.

Noble, D. (2009). The Music of Life: Biology Beyond Genes. Oxford: Oxford University Press.

Quinn, T. A., Granite, S., Allessie, M. A., Antzelevitch, C., Bollensdorff, C., Bub, G., Burton, R. A. B., Cerbai, E., Chen, P. S., Delmar, M., Difrancesco, D., Earm, Y. E., Efimov, I. R., Egger, M., Entcheva, E., Fink, M., Fischmeister, R., Franz, M. R., Garny, A., Giles, W. R., Hannes, T., Harding, S. E., Hunter, P. J., Iribe, G., Jalife, J., Johnson, C. R., Kass, R. S., Kodama, I., Koren, G., Lord, P., Markhasin, V. S., Matsuoka, S., McCulloch, A. D., Mirams, G. R., Morley, G. E., Nattel, S., Noble, D., Olesen, S. P., Panfilov, 
A. V., Trayanova, N. A., Ravens, U., Richard, S., Rosenbaum, D. S., Rudy, Y., Sachs, F., Sachse, F. B., Saint, D. A., Schotten, U., Solovyova, O., Taggart, P., Tung, L., Varró, A., Volders, P. G., Wang, K., Weiss, J. N., Wettwer, E., White, E., Wilders, R., Winslow, R. L., and Kohl, P. (2011). Minimum information about a cardiac electrophysiology experiment (MICEE): standardised reporting for model reproducibility, interoperability, and data sharing. Prog. Biophys Mol. Biol. 107, 4-10.

Qutub, A. A., MacGabhann, F., Karagiannis, E. D., Vempati, P., and Popel, A. S. (2009). Multiscale models of angiogenesis. IEEE Eng. Med. Biol. Mag. $28,14-31$.

Reumann, M., Fitch, B. G., Rayshubskiy, A., Keller, D. U., Weiss, D. L., Seemann, G., Dössel, O., Pitman, M. C., and Rice, J. J. (2008). Large scale cardiac modeling on the Blue Gene supercomputer. Conf. Proc. IEEE Eng. Med. Biol. Soc. 2008, 577-580.

Tanskanen, A., and Winslow, R. (2006). Integrative, structurally-detailed model of calcium dynamics in the cardiac diad. SIAM J. Multiscale Model. Simul. 5, 1280-1296.

Tanskanen, A. J., Greenstein, J. L., Chen, A., Sun, S. X., and Winslow, R. L. (2007). Protein geometry and placement in the cardiac dyad influence macroscopic prop- erties of calcium-induced calcium release. Biophys. J. 92, 3379-3396.

Travert, C., Jolivet, E., Sapin-de Brosses, E., Mitton, D., and Skalli, W. (2011). Sensitivity of patient-specific vertebral finite element model from low dose imaging to material properties and loading conditions. Med. Biol. Eng. Comput. doi: 10.1007/s11517-011-0825-0

Trayanova, N. A., and Rice, J. J. (2011). Cardiac electromechanical models: from cell to organ. Front. Physiol. 2:43. doi: 10.3389/fphys.2011.00043

Viceconti, M., Clapworthy, G., and Van Sint Jan, S. (2008). The virtual physiological human - a European initiative for in silico human modelling. J. Physiol. Sci. $58,441-446$.

Wang, Z., Gerstein, M., and Snyder, M. (2009). RNASeq: a revolutionary tool for transcriptomics. Nat. Rev. Genet. 10, 57-63.

Webster, D., and Müller, R. (2011). In silico models of bone remodeling from macro to nano-from organ to cell. Wiley Interdiscip. Rev. Syst. Biol. Med. 3, 241-251.

Winslow, R. L., and Greenstein, J. L. (2011). Cardiac myocytes and local signaling in nano-domains. Prog. Biophys. Mol. Biol. 107, 48-59.

Wood, L. D., Parsons, D. W., Jones, S., Lin, J., Sjoblom, T., Leary, R. J., Shen, D., Boca, S. M., Barber, T., Ptak, J., Silliman, N., Szabo, S., Dezso, Z., Ustyanksky, V.,
Nikolskaya, T., Nikolsky, Y., Karchin, R., Wilson, P. A., Kaminker, J. S., Zhang, Z., Croshaw, R., Willis, J., Dawson, D., Shipitsin, M., Willson, J. K., Sukumar, S., Polyak, K., Park, B. H., Pethiyagoda, C. L., Pant, P. V., Ballinger, D. G., Sparks, A. B., Hartigan, J., Smith, D. R., Suh, E., Papadopoulos, N., Buckhaults, P., Markowitz,S. D., Parmigiani, G., Kinzler, K. W., Velculescu, V. E., and Vogelstein, B. (2007). The genomiclandscapes of human breast and colorectal cancers. Science 318, 1108-1113.

Xu, Z., and Cai, X. (2008). Unbiased tau-leap methods for stochastic simulation of chemically reacting systems. J. Chem. Phys. 128, 154112.

Received: 13 October 2011; accepted: 13 October 2011; published online: 23 November 2011.

Citation: Winslow RL (2011) Grand challenges in computational physiology and medicine. Front. Physio. 2:79. doi: 10.3389/fphys.2011.00079

This article was submitted to Frontiers in Computational Physiology and Medicine, a specialty of Frontiers in Physiology. Copyright (๑) 2011 Winslow. This is an open-access article subject to a non-exclusive license between the authors and Frontiers Media SA, which permits use, distribution and reproduction in other forums, provided the original authors and source are credited and other Frontiers conditions are complied with. 